\title{
Neurodegeneration and the Gothenburg MCI study
}

Marie Eckerström, Anders Wallin

Institute of Neuroscience and Physiology, Sahlgrenska Academy, Sweden

https://doi.org/10.36505/ExLing-2019/10/0017/000379

\begin{abstract}
Mild cognitive impairment (MCI) is the stage between the expected cognitive decline of a normal aging population and the more serious decline of dementia. Typically, the performance of a cognitive test is considered impaired if it is $=>1.5$ standard deviations below normal controls. The ongoing Gothenburg mild cognitive impairment (MCI) study is an attempt to conduct longitudinal in-depth phenotyping of patients with different forms and degrees of cognitive impairment using various assessment tools. This paper presents the background of the linguistic and extra-linguistic parameters for early detection of cognitive impairment-project, a research which is partly based on data acquired at the Gothenburg MCI-study.
\end{abstract}

Key words: neurodegeneration, mild cognitive impairment, dementia

\section{Introduction}

Neurodegeneration is a progressive loss of the structure and function of neurons, eventually leading to neuron death. Some of the most common neurodegenerative disorders include Alzheimer's dementia (AD), frontotemporal dementia (FTD) and its variants, progressive supranuclear palsy (PSP), corticobasal degeneration (CBD), Parkinson's disease (PD), dementia with Lewy bodies (DLB), multiple system atrophy (MSA), and Huntington's disease (HD) (Erkkinen et al., 2018). Common factors of these diseases are that they gradually progress, and that there is to date no cure. The aetiology of the neuron death varies between diseases - it may be caused by tumours, vascular incidents such as stroke, viruses, toxins or excessive alcohol exposure, or the cause may be unknown. Partly as a result of a globally increased life expectancy, the prevalence of neurodegenerative disorders is increasing (Heemels, 2016).

Mild cognitive impairment (MCI) is a central concept in dementia, and especially Alzheimer's disease dementia research. MCI is not a disease in itself, but is a term used to describe an intermediate phase between normal cognition and dementia, characterized by cognitive impairment that is more pronounced than expected in normal aging but not at the advanced level of dementia (Petersen et al., 1997). Subjective cognitive impairment (SCI) is a related concept, although the cognitive decline in SCI is merely self-reported and not possible to measure with neuropsychological tests. The hypothesis forming the basis for these concepts is that neurodegenerative dementia disorders start

ExLing 2019: Proceedings of $10^{\text {th }}$ International Conference of Experimental Linguistics, 25-27 September 2019, Lisbon, Portugal 
gradually, with a starting-point of neuropathological events decades before clear symptoms appear (preclinical phase), a first symptomatic phase of several years when the change is only detectable by the affected individual (SCI), a second symptomatic phase of several years when cognitive changes are still subtle but has become possible to detect using tests and also noticeable by others (MCI), followed by the dementia stage in which cognitive decline is at a level that reduces the possibility to live an independent life.

\section{The Gothenburg MCI study}

The Gothenburg mild cognitive impairment (MCI) study started in 1999, and is a single-center clinical-pathophysiologic study aimed at investigating early and manifest phases of $\mathrm{AD}$ and vascular dementia $(\mathrm{VaD})$ in patients seeking medical care for their cognitive decline (Wallin et al., 2016). The clinical setting for the study is an outpatient specialised memory clinic in the Gothenburg region in the south-west part of Sweden. The catchment area includes approximately 1 million residents. Patients are mainly referred to the clinic from primary care level, in cases when a dementia disorder is suspected but when the methods of basic examination in the primary care is not sufficient for diagnosis. As of 2019, 781 patients and 124 healthy controls were included in the study, and 233 converted to dementia.

One of the central objectives of the study is to contribute to a better foundation for future treatment options by enhancing the knowledge of disease processes that may cause cognitive impairment, using multimodal markers. One important step is to improve the characterization of the early and manifest phases of different dementia disorders. For this purpose, single and multimodal measurements from the clinical-, neuropsychological-, genetic-, biochemistry-, neurochemistry, and neuroimaging fields are employed (Wallin et al., 2016).

Neuropsychological tests are the gold standard to measure cognitive functioning, and is therefore central in dementia research. The neuropsychological test battery used in the Gothenburg MCI study includes 20 tests related to the cognitive domains of learning/memory; speed/attention, language function; visuospatial function and executive function.

\section{Current $\sigma a t u s$ and the $\varphi$ ture of the Gothenburg MCI}

To date, several publications have been based on the neuropsychological tests in the Gothenburg MCI study. In the first years of the study, neuropsychological measures were analysed in 'broad strokes' using total scores and composite scores. The results generally highlight the importance and validity of neuropsychological measures in dementia research. To summarize key publications, we reported that: SCI/MCI is a highly heterogeneous diagnostic entity with significant impairments in all cognitive domains (Nordlund et al., 2005); SCI/MCI subjects with vascular disease performed worse on tests measuring speed/attention, visuospatial function, and executive 
function (Nordlund et al., 2007); SCI/MCI with AD markers performed overall worse, especially on tests of speed/attention and episodic memory (Nordlund et al., 2008); a combination of vascular disease and AD biomarkers was associated with executive impairment (Nordlund et al., 2011); memory, visuospatial, and language symptoms characterized incipient AD (Nordlund et al., 2010); executive and speed/attention symptoms characterized incipient SVD (Nordlund et al., 2010); and neuropsychological tests were the best predictors of dementia with a combination of markers further improving the predictive ability (Eckerström et al., 2013).

The collaboration with Dr. Dimitrios Kokkinakis and colleagues within the project 'Linguistic and extra-linguistic parameters for early detection of cognitive impairment' (Kokkinakis et al., 2016) is an important next step to further refine the study of cognitive functioning in relation to dementia progression, using novel technical and analytical tools other than traditional neuropsychological testing; see for instance Fraser et al. (2017); Themistocleous et al. (2018) and Linz et al., (2019).

\section{References}

Eckerström, C., Olsson, E., Bjerke, M., Malmgren, H., Edman, A., Wallin, A., Nordlund, A. 2013. A Combination of Neuropsychological, Neuroimaging, and Cerebrospinal Fluid Markers Predicts Conversion from Mild Cognitive Impairment to Dementia. J of Alzheimer's Disease 36. 421-431. DOI 10.3233/JAD-122440.

Erkkinen, M.G., Kim, M.O., schwind, M.D. 2018. Clinical Neurology and Epidemiology of the Major Neurodegenerative Diseases. Cold Spring Harb Perspect Biol. 10 (4). pii: a033118. doi: 10.1101/cshperspect.a033118.

Fraser, KC., Lundholm Fors, K., Kokkinakis, D., Nordlund, A. 2017. An analysis of eye-movements during reading for the detection of mild cognitive impairment. Conference on Empirical Methods in Natural Language Processing (EMNLP). Pp. 1016-1026. Copenhagen, Denmark

Heemels, M-T. 2016. Neurodegenerative disease. Nature. 10;539(7628):179. doi: 10.1038/539179a.

Kokkinakis, D., Lundholm Fors, K., Nordlund, A. 2016. Data Resource Acquisition from People at Various Stages of Cognitive Decline - Design and Exploration Considerations. 7th International Workshop on Health Text Mining and Information Analysis (LOUHI). An EMNLP Workshop. Texas, USA.

Linz, N., Lundholm Fors, K., Lindsay, H., Eckerström, M., Alexandersson, J., kkinakis, D. 2019. Temporal Analysis of Semantic Verbal Fluency Tasks in Persons with Subjective and Mild Cognitive Impairment. Sixth Workshop on Computational Linguistics and Clinical Psychology: Reconciling Outcomes. Minneapolis, USA.

Nordlund, A., Rolstad, S., Hellström, P., Sjögren, M., Hansen, S., llin, A. 2005. The Goteborg MCI study: mild cognitive impairment is a heterogeneous condition. J Neurol Neurosurg Psychiatry. 76(11):1485-90.

Nordlund, A., Rolstad, S., Klang, O., Lind, K., Hansen, S., llin, A. 2007. Cognitive profiles of mild cognitive impairment with and without vascular disease. Neuropsychology. 21(6):706-12. PMID: 17983284. 
Nordlund, A., Rolstad, S., Klang, O., Lind, K., Pedersen, M., Blennow, K., Edman, A., Hansen, S., llin A. 2008. Episodic memory and speed/attention deficits are associated with Alzheimer-typical CSF abnormalities in MCI. J Int Neuropsychol Soc. 14(4):582-90. doi: 10.1017/S135561770808079X.

Nordlund, A., Göthlin, M., llin, A. 2011. Vascular disease, Alzheimer's disease biomarkers and cognition in mild cognitive impairment: additive or synergetic effects? Dement Geriatr Cogn Disord. 32(4):250-6. doi: 10.1159/000334653.

Nordlund, A., Rolstad, S., Göthlin, M., Edman, A., Hansen, S., llin, A. 2010. Cognitive profiles of incipient dementia in the Goteborg MCI study. Dement Geriatr Cogn Disord. 30(5):403-10. doi: 10.1159/000321352.

Petersen, R.C., Smith, G.E., Waring, S.C., Ivnik, R.J., Kokmen, E., ngelos, E.G. 1997. Aging, memory, and mild cognitive impairment. Int Psychogeriatr. 9 Suppl 1:65-9.

Themistocleous, C., Eckerström, M., kkinakis, D. 2018. Diagnosis of Mild Cognitive Impairment from Speech in Swedish using Deep Sequential Neural Networks. Frontiers in Neurology, section Neurodegeneration. doi: 10.3389/fneur.2018.00975.

Wallin, A., Nordlund, A., Jonsson, M., Lind, K., Edman, A., Göthlin, M., Stålhammar, J., Eckerström, M., Kern, S., Börjesson-Hanson, A., Carlsson, M., Olsson, E., Zetterberg, H., Blennow, K., Svensson, J., Öhrfelt, A., Bjerke, M., Rolstad, S., kerström, C. 2016. The Gothenburg MCI study: Design and distribution of Alzheimer's disease and subcortical vascular disease diagnoses from baseline to 6year follow-up. J Cereb Blood Flow Metab. 36(1):114-31. 